\title{
Retrosigmoid Transmeatal Approach for Resection of Acoustic Neuroma: Operative Video and Technical Nuances of Subperineural Dissection for Facial Nerve Preservation
}

\author{
James K. Liu ${ }^{1,2}$ Vincent N. Dodson ${ }^{1}$ Robert W. Jyung ${ }^{2}$ \\ ${ }^{1}$ Departments of Neurological Surgery, Center for Cerebrovascular \\ and Skull Base Surgery, Rutgers University, New Jersey Medical \\ School, Neurological Institute of New Jersey, RWJ Barnabas Health, \\ Livingston and Newark, New Jersey, United States \\ 2 Otolaryngology/Head-and-Neck Surgery, Center for \\ Cerebrovascular and Skull Base Surgery, Rutgers University, New \\ Jersey Medical School, Neurological Institute of New Jersey, RWJ \\ Barnabas Health, Livingston and Newark, New Jersey, United States
}

\begin{abstract}
Address for correspondence James K. Liu, MD, Department of Neurological Surgery, Rutgers University, New Jersey Medical School, 90 Bergen Street, Suite 8100, Newark, NJ 07103, United States (e-mail: james.liu.md@rutgers.edu).
\end{abstract}

J Neurol Surg B 2019;80(suppl S3):S269-\$270.

The retrosigmoid (suboccipital) approach is the workhorse for most acoustic neuromas in the cerebellopontine angle. In this operative video atlas manuscript, the authors demonstrate the nuances of the subperineural dissection technique for microsurgical resection of an acoustic neuroma via the retrosigmoid transmeatal approach. The plane is developed by separating the perineurium of the vestibular nerve away from the tumor capsule. This perineurium provides a protective layer between the tumor capsule and the facial nerve which serves as a buffer to avoid direct dissection and potential trauma to the facial nerve. Using this technique during extracapsular tumor dissection helps to maximize the extent of tumor removal while preserving facial nerve function. A gross total resection of the tumor was achieved, and the patient exhibited normal facial nerve function (-Fig. 1). In summary, the retrosigmoid transmeatal approach with the use of subperineural dissection are important strategies in the armamentarium for surgical management of acoustic neuromas with the goal of maximizing tumor removal and preserving facial nerve function (-Fig. 2). The link to the video can be found at: https://youtu.be/L3IPtSvJt60.
Conflict of Interest

None.

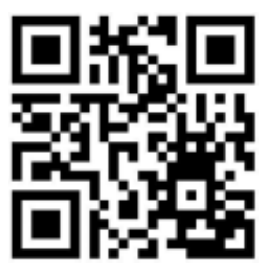

received

March 17, 2019

accepted

March 25, 2019

published online

April 30, 2019 www.thieme.com/skullbasevideos

www.thieme.com/jnlsbvideos
(C) 2019 Georg Thieme Verlag KG Stuttgart · New York

License terms

10.1055/s-0039-1688488. ISSN 2193-6331. 


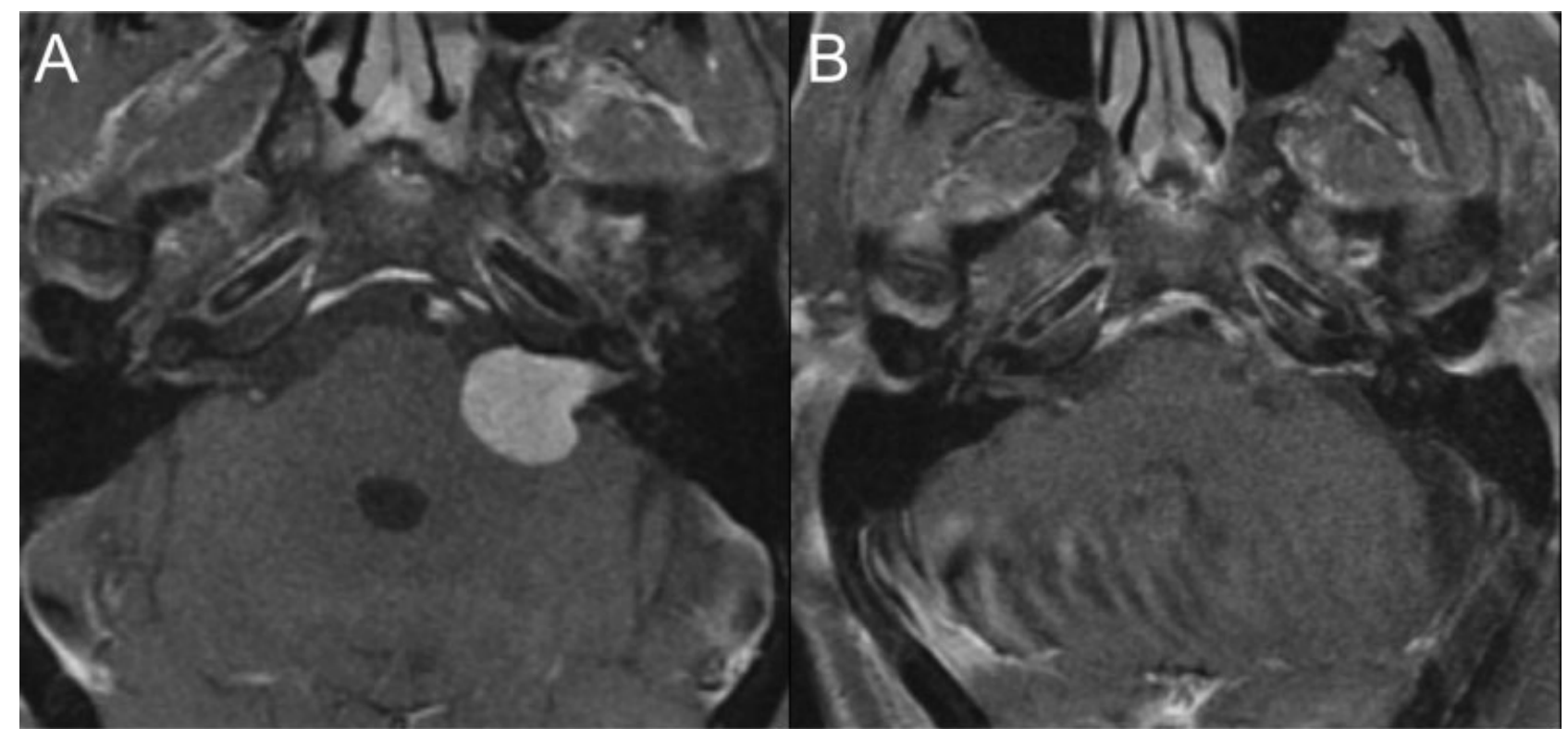

Fig. 1 (A) Preoperative axial T1-MRI with gadolinium demonstrates a Koos's grade 4 acoustic neuroma with brainstem compression. (B) Postoperative T1-MRI demonstrates successful gross total resection and relief of brainstem compression. MRI, magnetic resonance imaging.

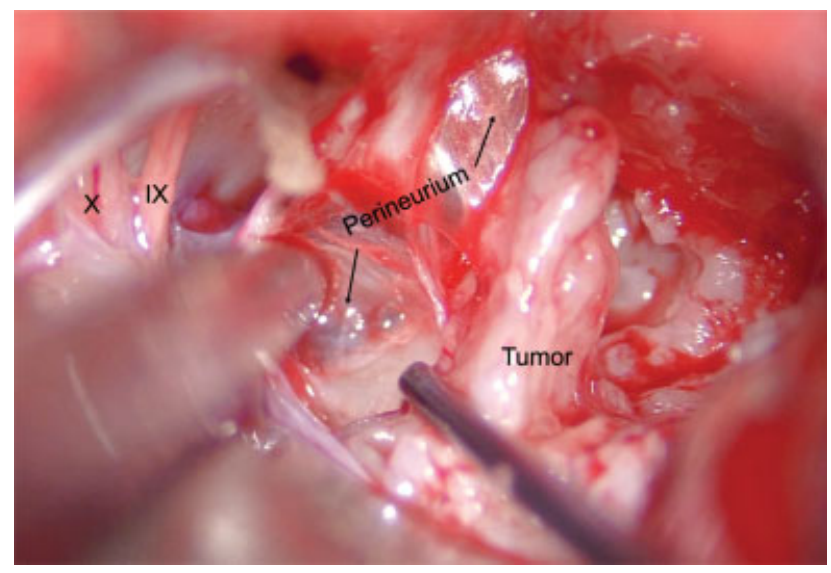

Fig. 2 Intraoperative photograph displaying the subperineural plane of dissection between the perineurium of the vestibular nerve and the tumor. 